ISSN: 2238-8052

\title{
AGROECOLOGIA E SVILUPPO RURALE NELLA REGIONE ORIENTALE DEL MINAS GERAIS
}

\author{
AGROECOLOGIA E DESENVOLVIMENTO RURAL NA REGIÃO ORIENTAL DO \\ ESTADO DE MINAS GERAIS
}

Andrea PRONTI ${ }^{1}$

Artigo recebido em 30/09/2017 e aceito em 27/11/2017

Palavras-chave:

Agroecologia

Projeto de

cooperação

Economia regional

Desenvolvimento

Brasil

Itália

Parole chiavi:

Agroecologia

Progetto di

cooperazione

Economia regionale

Sviluppo sostenibile

Brasile

Italia

\begin{abstract}
R E S U M O
Na região leste de Minas Gerais, realizou-se o projeto de cooperação internacional entre a Itália e o Brasil intitulado "Agroecologia e formação socioambiental para o desenvolvimento sustentável" com o objetivo de apoiar a agroecologia para o desenvolvimento rural local. A área é caracterizada pela produção extensiva de café, principalmente no âmbito da agricultura familiar. 0 café, além de sustentar quase que totalmente a economia local, é um dos principais impulsionadores da destruição da Mata Atlântica, um bioma local muito importante para a prestação de serviços ambientais. Graças à colaboração entre a Universidade de Turim e a RE.TE. Ong, no âmbito do projeto Uni.Coo, foi realizado um estudo econômico para comparar o uso de práticas agroecológicas e convencionais com o objetivo de verificar se o primeiro poderia realmente contribuir para o desenvolvimento sustentável da economia regional. Várias variáveis econômicas e ambientais foram comparadas em 6 unidades de produção. Os resultados do estudo indicam que as práticas agroecológicas podem fornecer, em média, rendimentos mais altos, melhor remuneração por trabalho, diversificação de renda e dietas, e que contribuem tanto para a redução do uso de produtos químicos quanto para a conservação da floresta. 0 estudo sugere que a agroecologia poderia representar um modelo possível de desenvolvimento agrícola sustentável para a região.
\end{abstract}

\section{RIASSUNTO}

Nell'area orientale dello stato del Minas Gerais è stato realizzato il progetto di cooperazione internazionale tra Italia e Brasile "Agroecologia e formazione socio ambientale per lo sviluppo sostenibile" con lo scopo di sostenere l'agroecologia per lo sviluppo rurale locale. L'area è caratterizzata dalla produzione estensiva di caffè principalmente a livello di agricoltura familiare. Il caffè, oltre a sostenere quasi totalmente l'economia locale, rappresenta uno dei maggiori driver di distruzione della Foresta Atlantica, bioma locale molto importante per la fornitura di servizi ambientali. Grazie alla collaborazione tra Università di Torino e RE.TE. Ong, nell'ambito del progetto Uni.Coo, è stato realizzato uno studio economico per confrontare l'utilizzo di pratiche agroecologiche e convenzionali con lo scopo di verificare se le prime potessero effettivamente contribuire allo sviluppo sostenibile dell'economia regionale. Sono state confrontate diverse variabili economiche e ambientali in 6 unità produttive. I risultati dello studio indicano che le pratiche agroecologiche siano in grado di fornire mediamente maggiori redditi, remunerare maggiormente il lavoro, diversificare i redditi e le diete, oltre a contribuire sia alla riduzione dell'uso prodotti chimici che alla conservazione forestale. Lo studio suggerisce che l'agroecologia possa rappresentare un possibile modello di sviluppo agricolo sostenibile per la regione.

\footnotetext{
${ }^{1}$ CNR-IRCRES, Consiglio Nazionale delle Ricerche, Istituto di Ricerca sulla Crescita Economica Sostenibile, Via Real Collegio 30, Moncalieri (TO). E-mail: andrea.pronti@ircres.cnr.it.
} 


\section{INTRODUZIONE}

L'agroecologia può essere definita come l'applicazione delle scienze ecologiche e dei loro principi allo studio, progettazione e gestione di agroecosistemi sostenibili, con lo scopo di renderli meno dipendenti da input esterni attraverso l'utilizzo delle sinergie biologiche dell'ecosistema in cui si inserisce l'attività agricola (Altieri e Nicholls, 2005; Gliessman, 1990). Le tecniche di produzione agroecologica si basano sulla consociazione di diverse colture e sul riutilizzo delle risorse create all'interno dell'unità agricola con lo scopo di creare un "agroecosistema", utilizzando l'ambiente locale in cui è inserita l'attività produttiva come parte integrante del sistema produttivo (Altieri, 1991). Le pratiche agroecologiche prevedono la massima diversificazione produttiva e la minimizzazione della dipendenza dell'agroecosistema dall'utilizzo di input chimici e lavoro con lo scopo di migliorare la produttività e la resistenza e favorire la rigenerazione dei suoli e della biodiversità (Altieri, 2002).

Tali pratiche consentono di migliorare le condizioni socio-economiche in situazioni di bassa disponibilità di capitali e molti risultati positivi ne hanno dimostrato la validità (Pretty et al., 2006; Altieri e Toledo, 2011). Al contempo l'agroecologia è in grado di ridurre le pressioni ambientali e l'inquinamento derivanti dall'agricoltura (Altieri, 2002). Le pratiche agroecologiche sono state anche riconosciute a livello internazionale come miglior sistema per sostenere lo sviluppo agricolo sostenibile a livello globale e ridurre le condizioni di povertà in cui vivono quasi un miliardo di persone residenti in aree rurali (Ifad, 2011; Fao, 2015; De Schutter, 2010).

Questo articolo intende esplorare tali tematiche attraverso un caso studio realizzato su alcuni piccoli produttori di caffè in Brasile, paese in cui tematiche legate alle disuguaglianza e alla povertà rurale è all'ordine del giorno.

Nell'ambito del progetto di ricerca Uni.Coo dell'Università degli studi di Torino e la Ong RE.TE. è stato realizzato uno studio di campo durante le attività di un progetto di cooperazione allo sviluppo nella Regione rurale Leste dello stato di Minas Gerais (MG) in Brasile. La ricerca è stata realizzata fra il 2012 e il 2014 nei comuni di Simonesia, Sao Joao do Manhuaçu, Caratinga e Manhuaçu, concentrandosi sul confronto socio-economico ed ambientale delle pratiche agricole agroecologiche e convenzionali utilizzate nella produzione di caffè nell'area di intervento del progetto (Fig. 1). Questo studio è figlio di una forte collaborazione tra Università, Ong e movimenti rurali e vuole essere un piccolo passo iniziale di approfondimento in studi economico-sociali relativi alla conoscenza dell'agroecologia come pratica agricola sostenibile. In questo articolo si intende analizzare l'agroecologia come possibile alternativa sostenibile alle pratiche monoculturali per i piccoli produttori della regione di studio, considerando la sostenibilità rispetto alla dimensione sociale, economica e ambientale. 
Figura 1: Mappa della Regione Orientale del Minas Gerais, area dove è stato realizzato lo studio.

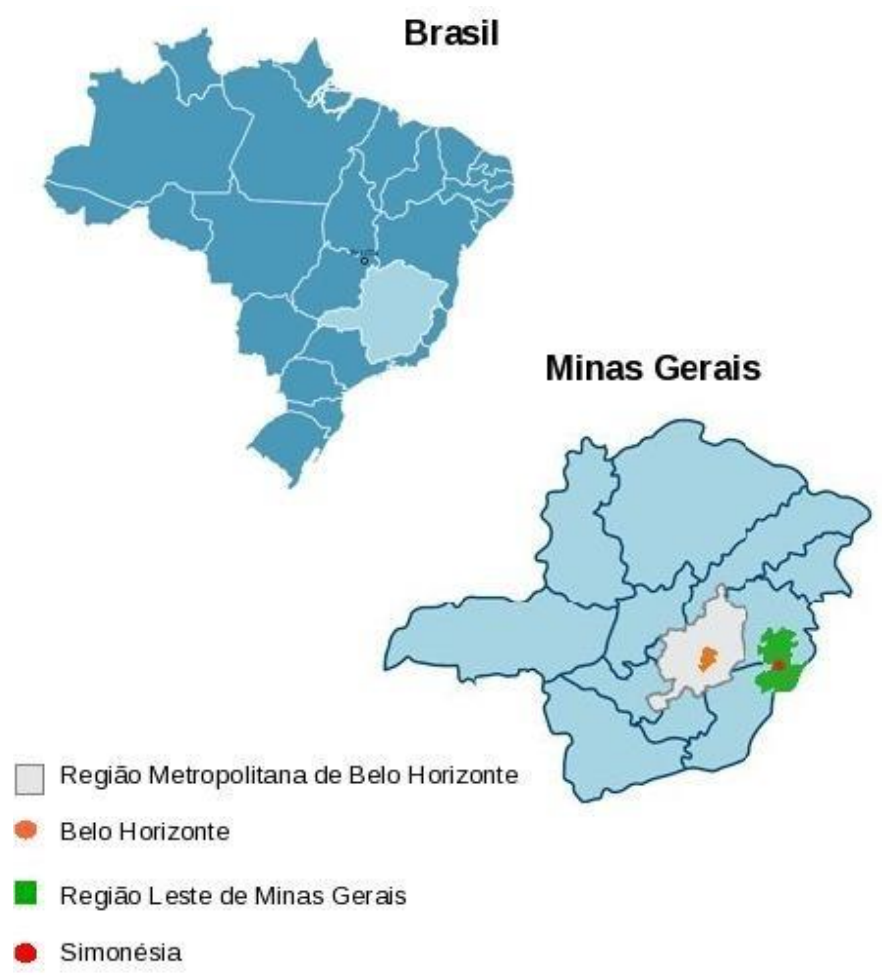

Fonte: Immagine fornita dalla Ong Rede de Intercâmbio de Tecnologias Alternativas.

\section{IL PROGETTO DI COOPERAZIONE}

Nell'ultimo decennio tra una minoranza di agricoltori dell'area di studio si sono diffusi esperimenti di coltivazione agroecologica introdotti da movimenti rurali e Ong. Grazie alla ottima facilità di adattamento e alle condizioni socio-economiche locali e alla facilità di trasmissione delle conoscenze per vie orizzontali, tali pratiche hanno iniziato a diffondersi negli anni con un discreto successo.

Il progetto di cooperazione internazionale "Agroecologia e formazione socio ambientale per lo sviluppo sostenibile della Regione Est di Minas Gerais - Brasil” realizzato dalle Ong RE.TE. (Italia) e REDE de Intercambio de Tecnologias Alternativas (Brasile) e sostenuto dal Ministero degli Affari Esteri della Repubblica Italiana prevedeva proprio come principale obiettivo di sostenere i processi agroecologici in atto nell'area per rafforzare il contributo dell'agricoltura familiare nello sviluppo sostenibile regionale. Il progetto ha sostenuto la diffusione dell'utilizzo di pratiche agroecologiche tramite canali di trasmissione informazione e formazione orizzontale, utilizzando il modello Campesino a Campesino (CAC) già ampiamente collaudato in tutta l'America Latina per attività di sviluppo rurale (Holt-Gimènez, 2008). I gruppi coinvolti sono stati principalmente gruppi formali e informali di agricoltori familiari e principalmente sindacati locali, associazioni di produzione e 
lavorazione di prodotti agricoli, cooperative di piccoli agricoltori e singole famiglie di agricoltori. Beneficiari del progetto sono state 182 famiglie di agricoltori sparse in 30 comunità. Durante le attività sono stati svolti vari workshop tenuti da operatori esperti, accademici o agronomi su tecniche di gestione agricola sostenibile relativi a rotazioni di cultivar, policoltivi, Sistemi Agro Forestali (SAF), conservazione idrica, gestione sostenibile dei suoli, metodi naturali di fertilizzazione e lotta a infestanti e parassiti. Altri eventi formativi sono stati relativi a diversificazione produttiva per il sostegno del reddito attraverso l'introduzione di piccoli animali, orti e frutta; e metodi di lavorazione e conservazione dei prodotti per dare valore aggiunto alla vendita dei prodotti. Le conoscenze acquisite dai beneficiari primari del progetto sono state diffuse all'interno delle comunità stesse attraverso l'approccio bottom-up CAC dove gli agricoltori formati sono divenuti essi stessi agenti di formazione e di trasmissione di saperi, in modo tale da consentire una diffusione delle pratiche agroecologiche ad ampio raggio ottimizzando le risorse disponibili per le attività di progetto.

Le azioni del progetto hanno anche sostenuto cooperative e organizzazioni rurali locali con l'obiettivo di rafforzare le capacità nella trasformazione di prodotti alimentari e la vendita sui locali. Per dare sostenibilità intergenerazionale alle azioni è stata sostenuta anche la scuola agroecologica "Escola Familia Agricola EFA Margarita Alves" con lo scopo di ridurre la dispersione scolastica e di consentire capacità utili alle famiglie degli studenti evitando migrazioni nelle aree urbane.

Il territorio abbracciato dal progetto fa parte del bioma Mata Atlântica che si estende su un'area equivalente a $1.315 .460 \mathrm{~km} 2$ e che originariamente si copriva la superficie di 17 stati del territorio Brasiliano. Attualmente ne rimane un'area pari al 7,91\% della copertura originale, contando le aree di foresta superiori a $100 \mathrm{HA}$, e pari all'11\% contando i frammenti superiori a $3 \mathrm{HA}$ (Sos Mata Atlântica, 2012). La Mata Atlântica è uno fra gli ecosistemi terrestri con maggiore complessità e biodiversità al mondo e fornisce importanti funzioni ecologiche tra cui regolazione climatica, conservazione del ciclo idrico e di nutrienti, conservazione della biodiversità, riduzione delle attività erosive e assorbimento di carbonio (Silvano et al, 2005). Tali funzioni ecologiche forniscono servizi ambientali fondamentali come impollinazione di frutta, stabilità climatica, fertilità dei suoli, produzione di ossigeno e fornitura di servizi idrici (Costanza et al., 1997). L'alta pressione antropica esercitata sulla Mata Atlântica incide fortemente sulla sua capacità di fornire questi servizi fondamentali alla vita. Una delle maggiori cause di frammentazione e deforestazione nella Regione è proprio la monocultura di caffè.

Il progetto ha sostenuto attività di riforestazione di piante native in aree agricole tentando di ridurre la perdita di biodiversità in atto nella regione e migliorare le condizioni ambientali attraverso il recupero di aree degradate e fonti idriche. Sono stati creati vivai con alberi nativi e da frutta per sostenere l'uso di SAF e ridurre la pressione antropica sul Corridoio ecologico Simonesia - Caratinga, 
habitat di molte specie faunistiche a rischio di estinzione come il Muriqui do Norte (Brachyteles hypoxanthus).

\section{IL CONTESTO SOCIO-ECONOMICO LOCALE}

La regione Est di MG è caratterizzata da una forte preponderanza dell'agricoltura familiare nella produzione agricola che rappresenta circa l' $88 \%$ di tutte le unità produttive agricole dello stato (IBGE, 2006).

L'agricoltura familiare brasiliana nonostante contribuisca fortemente alla produzione alimentare nazionale e alla produzione di commodities del paese è caratterizzata da basso accesso a capitali e credito e una limitata disponibilità di terra (IBGE, 2006). In questo tipo di conduzioni la creazione di reddito deriva quasi totalmente da attività agricole, il coinvolgimento di lavoro familiare è presente in tutte le fasi di produzione, lavorazione e distribuzione dei prodotti agricoli (Pimbert, 2008).

L'economia dei quattro comuni analizzati dipende quasi esclusivamente dalla produzione di caffè, ponendo quindi gran parte degli abitanti della regione in una posizione di vulnerabilità rispetto alla volatilità dei prezzo internazionali. I comuni considerati nello studio sono prevalentemente agricoli, circa il 60\% della popolazione è occupata nel settore primario e la struttura fondiaria della zona è caratterizzata dalla predominanza della piccola proprietà (fino a $10 \mathrm{Ha}$ ). La prevalenza dell'agricoltura familiare nei quattro comuni è elevata, le unità produttive agricole a conduzione familiare in media sono pari all' $85 \%$ del totale, nel 2010 le conduzioni agricole familiari ammontavano a un totale di 5.899 unità contro le 1.046 di grandi produttori agricoli (IBGE, 2010; IBGE, 2006). Secondo un'analisi dei dati del censimento 2010 dell' Istituto Brasiliano di Geografia e Statistica (IBGE), la produzione locale totale era di 54 mila tonnellate di caffè, l'area coltivata era di circa 40 mila ettari, circa il 99\% dell'area coltivata totale, con un valore totale prodotto di 260 milioni di Reais (pari a circa 72 milioni di euro), pari in media al 97,2\% del valore della produzione totale dei quattro comuni considerando l'intera filiera di produzione (IBGE, 2010; IBGE, 2006).

Il Brasile è il maggiore produttore di caffè al mondo, in media tra il 1990 e il 2016, ha prodotto annualmente 38.4 mila sacche di caffè verde da $60 \mathrm{~kg}$ pari a circa il $32 \%$ di tutta la produzione mondiale (ICO, 2016). Lo stato di MG è quello a livello nazionale con la maggiore produzione pari al $51 \%$ della produzione totale brasiliana di caffè (MAPA, 2015), con un grande contributo da parte della piccola agricoltura familiare (IBGE, 2006).

Il settore del caffè è caratterizzato da una forte concentrazione lungo la filiera e nelle fasi successive al raccolto che condizionano i margini di reddito dei piccoli agricoltori (FA0, 2004; Vorley, 2003). Il mercato del caffè è anche caratterizzato da una grande variabilità di prezzo che crea diversi 
rischi per i piccoli produttori (ICO, 2002; ICO, 2003; ICO, 2004). Secondo l'International Coffee Organization ciò è dovuto da vari fattori esogeni completamente incontrollabili fra cui: produzione di altri paesi, economia e commercio internazionale, speculazioni finanziarie, prezzo di altre commodity quotate nelle borse internazionali, quantità di stock invenduti degli anni passati ed eventi climatici sfavorevoli (ICO, 2010; ICO, 2011a; ICO, 2011b). Per i piccoli produttori dipendere da un'unica coltura molto volatile come il caffè è rischioso, la raccolta e la vendita avvengono una sola volta durante l'anno ed il prezzo di vendita è di difficile previsione, riducendo le possibilità di creare reddito sicuro per le famiglie. Tali rischi e problemi non controllabili a livello locale si ripercuotono fortemente su tutto lo sviluppo economico della regione.

\section{LA RICERCA}

L'agroecologia è stata introdotta nell'area da alcuni agricoltori sensibili a tematiche ambientali e sociali venuti a contatto con i movimenti rurali e di sviluppo nazionali. La diversificazione dei redditi e il miglioramento delle condizioni produttive ed ambientali sono tra le priorità delle famiglie rurali. I buoni risultati ottenuti nel tempo da alcuni produttori hanno confermato la validità di tali pratiche a livello locale, e la trasmissione di conoscenza peer to peer tra agricoltori ha consentito l'estensione dell'uso di tali pratiche in buona parte della regione, seppur rimanendo un approccio marginalizzato rispetto alle tecniche convenzionali basate sull'utilizzo della monocoltura che caratterizzano la regione. Nonostante le coltivazioni di caffè siano molto adatte e all'uso di pratiche agroecologiche, in quanto le piantagioni tradizionalmente sono coltivate sotto ombreggiamento e strutturate come sistemi agroforestali (SAF) (Gliessman, 2008; Moguel e Toledo 1999; Perfecto et al. 2005), la maggioranza dei coltivatori (sia piccoli che grandi) ha comunque continuato ad utilizzare modalità produttive convenzionali con uso di caffè not-sun shaded per motivazioni apparentemente economiche e di produttività.

Oltre al miglioramento delle proprie condizioni socio-economiche, alcuni produttori di caffè hanno iniziato a convertirsi all'uso di pratiche agroecologiche con lo scopo di ridurre gli impatti ambientali derivanti dalle attività agricole convenzionali, introdotti durante la "Rivoluzione Verde", causa di forte pressione sulle risorse naturali locali. Nonostante le condizioni socio-economiche e ambientali dell'area possano sembrare favorevoli all'estensione su scala regionale dell'agroecologia, molti produttori locali hanno mostrato una bassa propensione alla transizione verso pratiche sostenibili principalmente perché spaventati dalla possibili perdite di reddito e produttività, nonostante ciò non sia sostenuto da nessuno studio o prova rilevante. 
Obiettivo dello studio è stato confrontare unità produttive che utilizzassero pratiche agricole agroecologiche e convenzionali nell'area del progetto, con lo scopo di stimare le performance socioeconomiche e ambientali dei due metodi produttivi.

Durante un periodo di ricerca sul campo di quattro mesi tra Aprile e Agosto 2012, sono state confrontate attraverso analisi nel dettaglio le attività di vari produttori locali, stimando e comparando la capacità di generare reddito rispetto alla volatilità del prezzo del caffè, le condizioni lavorative, la produttività, la suddivisione dei costi, il livello di diversificazione produttiva e l'impatto ambientale. In questo articolo vengono presi in considerazione sei produzioni familiari di cui quattro agroecologici e due convenzionali.

I dati sono stati raccolti tramite un questionario semi-strutturato durante lo svolgimento di interviste in profondità realizzate direttamente nelle unità produttive e attraverso varie visite di studio nelle proprietà. Sulla base dei dati dichiarati dagli agricoltori sono stati estrapolati gli aspetti economici rilevanti delle singole attività produttive. Tale procedura consente la confrontabilità dei risultati con quelli di altri studi similari realizzati sullo stesso tema (Donizette De Oliveira et al., 2000; Reichhuber e Requate, 2012).

Gli agricoltori intervistati sono tutti di tipo familiare con proprietà tra 1 e $8 \mathrm{Ha}$, di cui quattro agroecologici e due convenzionali. Come produttori agroecologici sono stati considerati quei produttori che avessero intrapreso un percorso di transizione verso pratiche sostenibili in agricoltura basate sui principi dell'agroecologia da almeno 10 anni.

L'analisi ha considerato tutto il valore generato dall'attività produttiva, sia quello derivante dalla produzione principale (il caffè) che quello connesso ad attività agricole secondarie o prodotti lavorati, anche in caso queste non assumessero valore diretto di mercato in quanto non commercializzate. Ciò è stato deciso per includere nella creazione di reddito prodotti importanti per l'autoconsumo familiare con lo scopo di non focalizzarsi in un'ottica esclusivamente produttivistica non adatta al contesto locale caratterizzato da forte povertà rurale, bassi redditi e scarsa sicurezza alimentare (Wojtkowski 2008). I valori di produzione e redditività in mancanza di registrazioni di bilancio sono stati stimati attraverso le dichiarazioni di produzione media da parte degli intervistati e valutati in base alle principali fonti di rilevamento di prezzi nazionali. I valori di costo e di ricavo sono stati stimati in base a riferimenti di mercato di agenzie pubbliche specializzate, associazioni di categoria, centri studi, rilevazioni locali e dichiarazioni degli intervistati. Per il caffè è stato utilizzato il prezzo in valuta Brasiliana (Real) nella città di San Paolo di una sacca da $60 \mathrm{~kg}$ di caffè verde, di qualità "Bebida Dura" al netto delle tasse, riferendosi ai prezzi giornalieri in valori nominali dal 03-01-2000 al 29-06-2012, forniti dal Centro de Estudos Avançados em Economia Aplicada (CEPEA).I prezzi di prodotti orticoli, frutticoli e produzione animale sono stati utilizzati i dati forniti da Centrais de Abastecimento de Minas Gerais (CEASAMINAS) e Companhia Nacional de Abastecimento (CONAB). Il 
prezzo di vendita di animali vivi da pecuaria.com, il valore della piscicoltura dai prezzi di CEAGESP. I prodotti secondari processati, materiali fertilizzanti e pesticidi i prezzi pubblicati da CONAB . Per gli elementi arborei da Centro Inteligencia em Floresta. Per il calcolo del costo del lavoro è stato utilizzato il valore orario di 3,925 R\$, in base al salario minimo mensile del 2012 stabilito dalla legge "LEI № 12.382, DE 25 DE FEVEREIRO DE 2011" del governo Brasiliano. In mancanza di dati su registri pubblici i prezzi non identificabili sono stati rilevati nei mercati locali.

\section{METODOLOGIA}

Sono state analizzate e confrontate la capacità di generare reddito di ogni unità produttiva presa in analisi simulando variazioni del prezzo di mercato del caffè, costruendo un'analisi costibenefici $(\mathrm{ACB})$ per ogni singola proprietà. Sono stati utilizzati tre differenti prezzi del caffè per stimare la resilienza economica delle singole attività rispetto a tre scenari differenti di prezzo e stimare così la capacità di creazione di valore economico in condizioni di rischio (Perman et al., 2003; Hanley e Barbier, 2009; Pearce et al., 2006). Sono stati utilizzati: un prezzo alto, utilizzando il prezzo massimo raggiunto nel mercato in 12 anni (555,19 R\$ per sacca); un prezzo basso, utilizzando quello minimo raggiunto in 12 anni (101,48 R\$); ed il prezzo medio di mercato degli ultimi 5 anni $(340,57 \mathrm{R} \$)$. Sono stati considerati tutti i benefici e i costi delle aziende analizzate considerando una stima di costi d'investimento iniziali, costi variabili e ricavi in base ai dati dichiarati dagli agricoltori. Considerando un arco temporale di 23 anni2 e tassi di sconto pari a 0,06; 0,08 e 0,1, per valutare i differenti risultati in base al costo opportunità in termini di potenziali investimenti alternativi (Perman et al., 2003; Hanley e Barbier, 2009; Pearce et al., 2006). Per semplicità è stato ipotizzato che tutte le singole produzioni iniziassero da zero, come se fossero tutte frutto di progetti iniziati lo stesso anno in $t=0$, imputando i costi d'investimento iniziali nell'anno zero per tutte le proprietà analizzate. Costi e ricavi relativi alla raccolta del caffè sono stati imputati solo da $t>1$, per tutti gli altri periodi sono stati considerati costi di manutenzione dell'area e ricavi relativi a caffè e produzioni secondarie come frutta e produzioni orticole, prodotti processati all'interno dell'azienda derivanti dalle produzioni e produzioni animali, prodotti arborei estratti dall'area produttiva e dalla foresta. Le produzioni agricole sono state stimate come quantità medie considerabili come produzioni usuali dell'azienda.

I valori ottenuti sono stati divisi per la superficie produttiva di ogni proprietà analizzata in modo tale da ottenere come unità di comparazione economica il singolo ettaro produttivo di ogni azienda.

\footnotetext{
2 L'arco temporale utilizzato è di 23 anni, in modo tale da consentire una stima di reddito sul medio-lungo periodo in vent'anni di produzione, in quanto i primi due anni successivi all'impianto il caffè è improduttivo e la prima produzione utile inizia il terzo anno.
} 
La Produttività per ettaro coltivato a caffè è stata stimata e confrontata per verificare se effettivamente i due approcci agricoli incidessero diversamente sui livelli di produttività. Tale valore è stato stimato in termini di quantità di sacche di caffè da $60 \mathrm{~kg}$ divise per il numero di ettari dedicati a tale produzione come indicatore dell'efficienza produttiva dei due approcci agricoli.

E' stata realizzata una stima dell'incidenza dei costi variabili sui ricavi in valore percentuale per confrontare se effettivamente le pratiche agroecologiche fossero in grado di ridurre l'incidenza dei costi operativi in modo tale da offrire maggiori margini di guadagno. Sono stati anche stimati i livelli dei costi legati agli input chimici utilizzati nelle unità produttive come fertilizzanti chimici, correttori di suolo e pesticidi, in termini percentuale rispetto ai costi totali di produzione.

Per il confronto sulle condizioni di lavoro offerte dai due approcci sono stati calcolati l'intensità di lavoro e la remunerazione del lavoro. E' stata confrontata anche la diversificazione produttiva delle singole proprietà con lo scopo di analizzare la dipendenza dei redditi generati dalle singole aree produttive come percentuale rispetto al reddito totale generato. Le aree produttive sono state suddivise in cash crop (caffè), produzioni secondarie (frutta, orticole e allevamento), prodotti lavorati e semilavorati creati all'interno dell'unità produttiva (marmellate, saponi, formaggi, ecc..) e area forestale (legname estratto per costruzioni o altri usi ). E' stato anche stimato il livello di agrobiodiversità di ogni singola unità produttiva come numero delle diverse componenti biologiche di rilevanza produttiva coltivate o allevate nella stessa.

E' stato confrontato l'impatto sulle risorse naturali delle due modalità produttive come inquinamento agricolo e conservazione di aree forestali. Il primo è stato stimato come quantitativo di input chimici utilizzati per ettaro produttivo, sono stati considerati tutti gli input inquinanti in $\mathrm{kg}$ utilizzati nell'area mediamente in ogni singola stagione produttiva. Sono stati suddivisi in fertilizzanti, correttori di suolo e pesticidi. La conservazione forestale è stata stimata come percentuale delle aree forestali presenti nell'unità produttive rispetto all'intera superficie disponibile.

\section{RISULTATI PRINCIPALI E COMMENTI}

I risultati dello studio indicano come le pratiche agroecologiche, nonostante riducano la produttività per ettaro, siano in grado di consentire maggiori livelli di reddito grazie principalmente alla riduzione dei costi generali di gestione.

I produttori agroecologici hanno ottenuto in linea generale risultati economici migliori rispetto ai produttori convenzionali. Nella generazione di reddito i produttori agroecologici hanno evidenziato VAN per ettaro superiori nello scenario con prezzi medi e di stabilizzare i redditi in presenza di cadute di prezzo. Infatti, nello scenario con prezzi bassi di caffè, i VAN degli agricoltori agroecologici rimangono positivi, mentre gli agricoltori convenzionali subiscono ingenti perdite, mostrando valori 
economici negativi (Figura 2). In questo scenario i produttori agroecologici mostrano VAN compresi tra 91 mila e 39 mila Real per ettaro, mentre i due convenzionali rispettivamente valori pari a 77.962,03 R \$/HA e -30.942,64 R \$/HA. Solo nello scenario con prezzi alti i produttori convenzionali non sembrano discostarsi eccessivamente dai produttori agroecologici, nonostante questi ultimi mostrino comunque valori di VAN mediamente superiori e pari a 198.796,68 R \$/Ha contro quello dei produttori convenzionali pari a $130.403,18 \mathrm{R} \$ \mathrm{Ha}$.

Figura 2: VAN per ettaro produttivo $(\mathrm{R} \$ / \mathrm{HA})$ di ogni produttore analizzato con tre differenti livelli di prezzo: prezzo medio 340,57 R\$/sacca; prezzo massimo 555,19 R \$/sacca; prezzo minimo 101,48 $\mathrm{R} \$ /$ sacca.

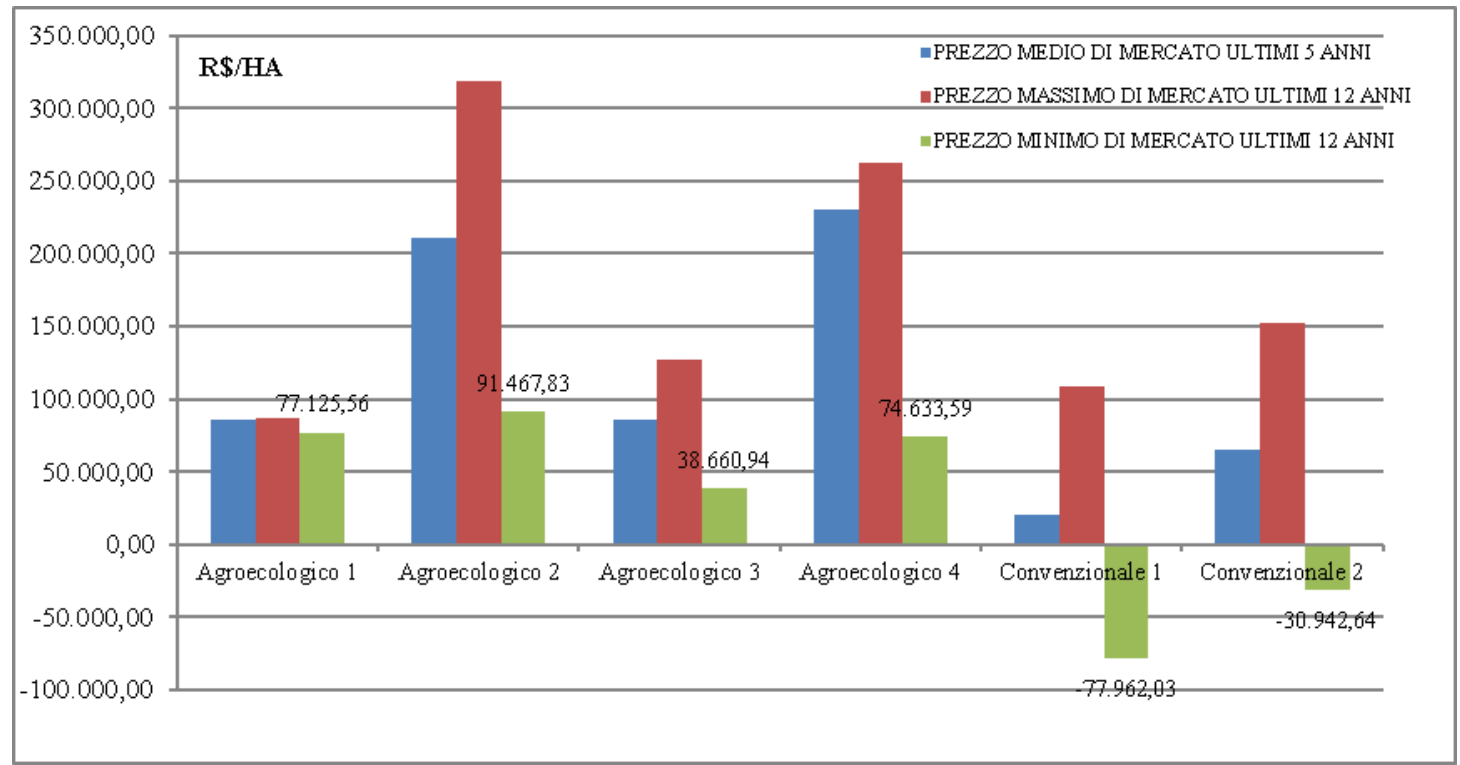

Fonte: Elaborazione dell'autore.

I produttori agroecologici mostrano livelli di produttività mediamente inferiori rispetto ai produttori convenzionali (Figura 3), ciò però non si riflette automaticamente in una maggiore generazione di redditi, infatti i ricavi maggiori dei produttori convenzionali derivanti da superiori livelli di produttività vengono erosi da costi di produzione elevati, principalmente lavoro, uso di macchinari e utilizzo di input di sintesi (fertilizzanti, pesticidi e correttori di suolo) (Figura 4). 
Figura 3: Produttività degli agricoltori analizzati in numero di sacche di caffè verde prodotte per ogni singolo ettaro coltivato a caffè.

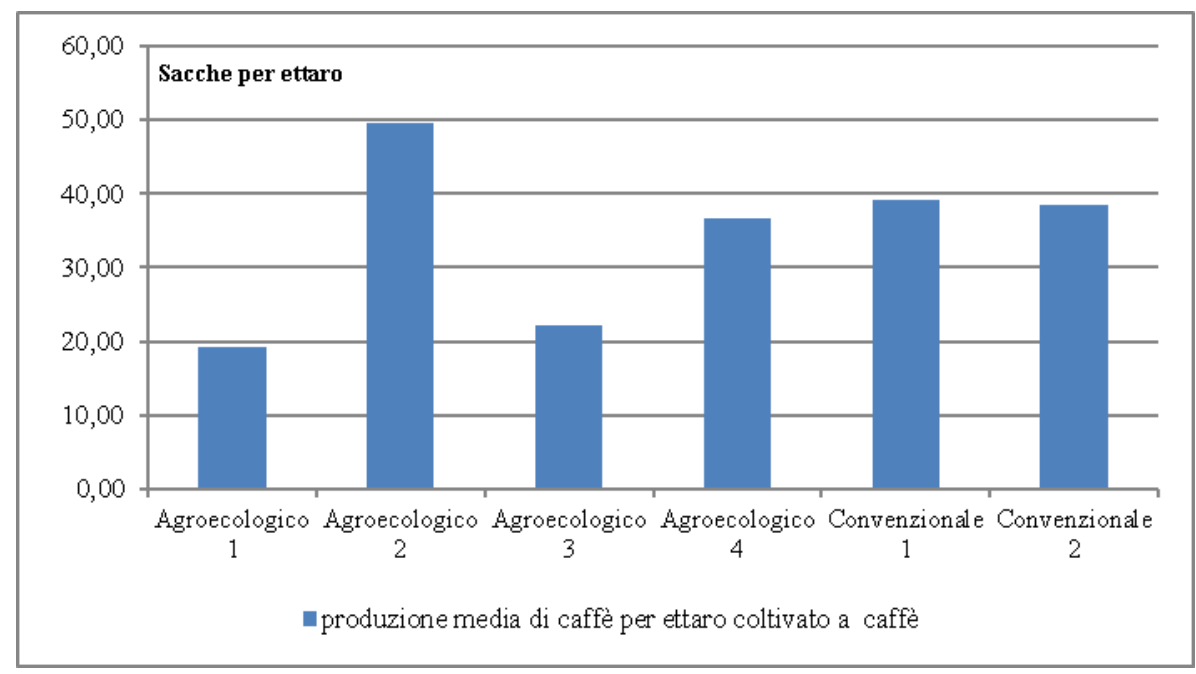

Fonte: Elaborazione dell'autore.

Figura 4: Costi variabili di produzione su ricavi totali e Costi relativi all'acquisto di input chimici rispetto ai costi totali sostenuti.

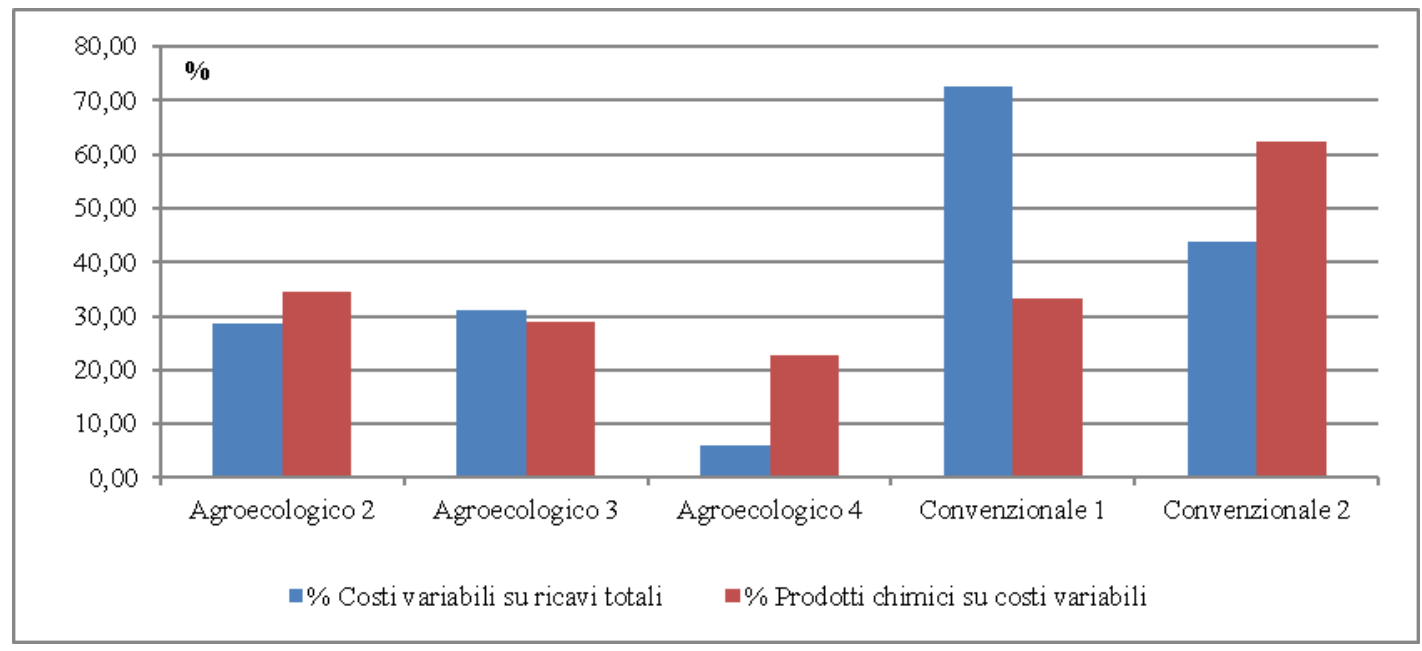

Fonte: Elaborazione dell'autore.

Rispetto all'intensità e alla remunerazione del lavoro le differenze tra i due approcci produttivi non appaiono così marcate, a parte in un caso in cui la remunerazione è nettamente superiore agli altri grazie alla creazione di un agroecosistema altamente produttivo e con bassa necessità d'intervento esterno. L'analisi del lavoro realizzata non è però in grado di confermare, o meno, una effettiva diversità nell'intensità di lavoro dei due metodi produttivi in quanto ogni singola unità produttiva adotta un sistema di lavoro e di gestione a se stante.

La stabilizzazione del reddito rispetto all'andamento del prezzo sembra dipendere principalmente dalla diversificazione produttiva ottenuta tramite l'applicazione delle pratiche 
agroecologiche tali da dissociare l'economia dell'unità produttiva dalla sola coltivazione di caffè. I produttori agroecologici ricavano reddito familiare da altre componenti produttive come orticole, frutta, allevamento e prodotti secondari lavorati e hanno un maggiore livello di agrobiodiversità tra 44 e 25 diverse specie allevate e coltivate contro 3 e 1 dei convenzionali (Figura 5).

Figura 5: Diversificazione produttiva in base alla generazione dei ricavi aziendali per ogni singola area produttiva (caffè, coltivazioni secondarie, prodotti secondari e area forestale) per produttori agroecologici e convenzionali.

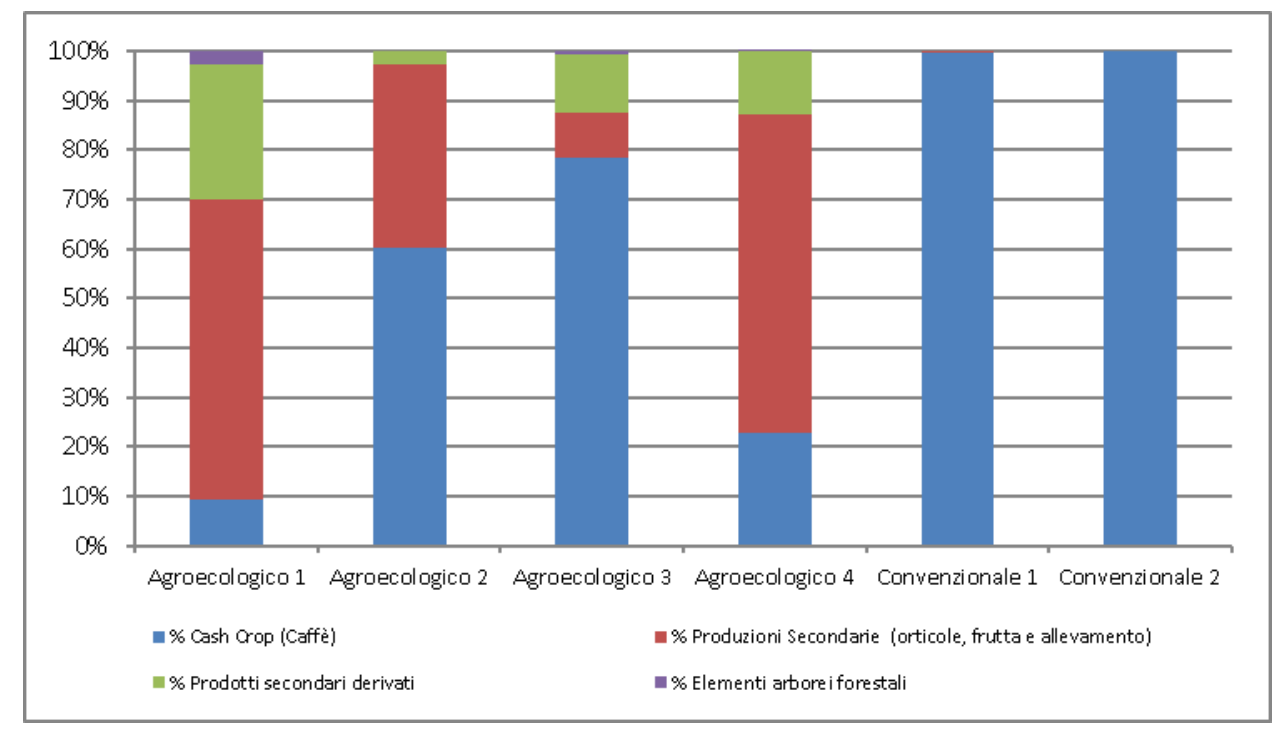

Fonte: Elaborazione dell'autore.

Rispetto all'impatto ambientale, le pratiche agroecologiche utilizzate dagli agricoltori coinvolti nello studio mostrano mediamente una netta riduzione nell'uso di input chimici per ettaro produttivo, nessun produttore agroecologico utilizza pesticidi chimici a differenza dei produttori convenzionali (Tabella 1). Oltre ad un minore inquinamento agricolo le pratiche agroecologiche sembrano consentire un maggiore livello di conservazione forestale rispetto alle pratiche convenzionali. I produttori analizzati mostrano livelli di conservazione mediamente superiori del $17 \%$ rispetto ai livelli di aree forestali conservate dai produttori convenzionali (Figura 8), i quali conservano solamente circa il 20\% del totale come richiesto per il codice forestale brasiliano dalla legge federale Lei 12.651/20123. Questi elementi confermano come l'uso dell' agroecologia possa contribuire alla conservazione di aree forestali in zone frammentate e sotto forte pressione antropica e alla riduzione dell'inquinamento agricolo (Altieri, 1991).

\footnotetext{
${ }^{3}$ La legge brasiliana prevede la conservazione di aree forestali nelle proprietà adibite ad agricoltura con lo scopo di ridurre l'effetto della deforestazione e conservare la biodiversità. Ogni macroregione ha una diversa richiesta minima di conservazione, per il MG la quota minima è il $20 \%$ del totale della superficie della proprietà agricola. Fonte: https://www.embrapa.br/codigo-florestal/area-de-reserva-legal-arl (ultimo accesso a Marzo 2017).
} 
Tabella 1: Utilizzo di input chimici e prodotti di sintesi in kg per ettaro produttivo per produttori agroecologici e convenzionali.

\begin{tabular}{llll}
\hline TIPO & $\begin{array}{l}\text { fertilizzanti } \\
\text { chimici } \\
\text { (Kg/HA) }\end{array}$ & $\begin{array}{l}\text { correttori } \\
\text { suolo } \\
\text { (Kg/HA) }\end{array}$ & $\begin{array}{l}\text { di } \\
\text { chimici } \\
\text { (Kg/HA) }\end{array}$ \\
\hline Agroecologico 1 & 62,50 & 109,38 & 0,00 \\
Agroecologico 2 & $2.419,35$ & $1.935,48$ & 0,00 \\
Agroecologico 3 & 616,44 & 821,92 & 0,00 \\
Agroecologico 4 & 171,67 & 833,33 & 0,00 \\
Convenzionale 1 & $2.370,87$ & $1.859,50$ & 17,43 \\
Convenzionale 2 & $2.243,50$ & $1.500,00$ & 2,69 \\
\hline
\end{tabular}

Fonte: Elaborazione dell'autore.

Figura 6: Conservazione di aree forestali in percentuale rispetto alla superficie totale presente nella proprietà per produttori agroecologici e convenzionali.

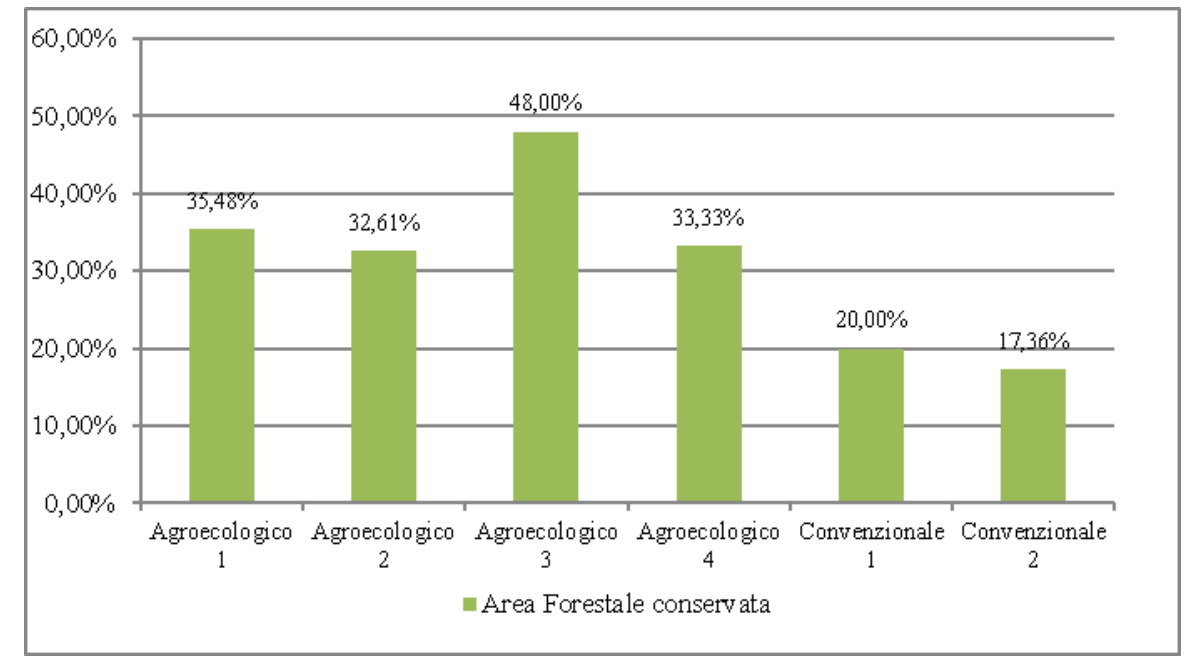

Fonte: Elaborazione dell'autore.

\section{CONCLUSIONI}

Secondo lo studio realizzato l'uso dell'agroecologia nella piccola agricoltura locale permette di migliorare sia le condizioni socio-economiche sia quelle ambientali.

Le pratiche agroecologiche migliorano la generazione di reddito e lo stabilizzano nel tempo svincolandolo dall'andamento del mercato del caffè, riducendo così il rischio di perdite in situazioni di mercato avverse. Ciò dipende principalmente da un maggiore livello di diversificazione produttiva, tale da garantire anche maggiori quantitativi di beni alimentari per la famiglia.

Nonostante la produttività sia mediamente inferiore per chi adotta pratiche sostenibili, i costi di produzione risultano inferiori soprattutto grazie alla riduzione di lavoro e materiali di sintesi. 
Le pratiche agroecologiche consentono di ridurre nettamente l'uso di fertilizzanti chimici, correttori di suolo e pesticidi rispetto alle modalità produttive convenzionali. Dal lato ambientale anche la conservazione di aree forestali è risultata superiore, e in alcuni casi doppia, rispetto al livello di conservazione ottenuto tramite l'uso di pratiche convenzionali.

L'agroecologia rispetto al caso studio sembra dare ottimi risultati e buone speranze per lo sviluppo sostenibile della regione Est di MG, infatti, nonostante il numero limitato di aziende analizzate, i risultati mostrano come le pratiche agroecologiche possano rappresentare una valida alternativa all'agricoltura convenzionale. L'uso maggiore di queste pratiche potrebbe migliorare fortemente le condizioni sociali e ambientali riducendo la dipendenza dell'economia locale dalla produzione del caffè riducendo quindi i rischi legati alle fluttuazioni di prezzo.

I risultati sono incoraggianti e indicano come le attività del progetto di cooperazione internazionale di sviluppo rurale considerato dallo studio possa effettivamente contribuire allo sviluppo sostenibile dell'intera regione.

\section{REFERENCE}

ALTIERI M. A., NICHOLLS C., 2005. "Agroecology and the Search for a Truly Sustainable Agriculture", Basic textbooks for environmental training, University of California, Berkeley (USA).

ALTIERI M. A., Toledo V., 2011. "The agroecological revolution in Latin America: rescuing nature, ensuring food sovreignty and empowering peasant", The Journal of Peasant Studies, vol. 38, n. 3, pp. 587-612.

ALTIERI M.A., 1991. Agroecologia. Prospettive scientifiche per una nuova agricoltura. Franco Muzzio \& C. Editore spa, Padova.

COSTANZA R., D'ARGE R., DE GROOT R., FARBERK S., GRASSO M., HANNON B., LIMBURG K., NAEEM S., O'NEILL R.V., PARUELO J., RASKIN R.G., SUTTONKK P., VAN DEN BELT M., 1997. "The value of the world's ecosystem services and natural capital". Nature (1997), Vol.387, pp. 253-260.

DE SCHUTTER O., 2010. "Report of the special Rapporteur on the right of food", United Nation General Assembly, Gen 2010. DONIZETTE DE OLIVERA A., Scolforo J.R, De Paula Silveira V., 2000."Analise Economica de um sistema agro-silvo-pastoril com eucalipto implantado em regiao de cerrado", Ciência Florestal, vol. 10, pp. 1-19.
FAO, 2004. "The state of agricultural commodity markets", Food and Agriculture Organization of the United Nation, Roma.

FAO, 2015. "The State of Food Insecurity in the World. Meeting the 2015 international hunger targets: taking stock of uneven progress". Food and Agriculture Organization of the United Nation, Roma.

GLIESSMAN S., 1990. "Agroecology:research the ecological basis for sustainable agriculture", Springer-Verlag New York Inc., Madison, Wisconsin(USA).

GLIESSMAN S., 2008. "Agroecological foundations for designing sustainable coffee agroecosystem", In Bacon M. et al., Confronting the coffee crisis. Fair trade, Sustainable livelihoods and Ecosystems in Mexico and Central America, The MIT Press, Massuchusetts Institute of Technology, 2008 Boston, (USA).

HANLEY N., BARBIER E.B, 2009. "Pricing Nature Cost-Benefit Analysis and Environmental Policy", Edward Elgar Publishing Limited.

HOLT-GIMẼNEZ E., 2008. "Campesino a Campesino. Voces de Latinoamérica. Movimiento Campesino a Campesino para la Agricultura Sustentable". Food First Book, Managua(Nicaragua). 
IBGE, 2006. "Ceso Agropecuario 2006.

Agricultura familiar. Primeiros resultados.

Brasil, Grandes Regioes e Unidades da federeçao", Instituto Brasileiro de Geografia e Estatistica, Brasilia.

IBGE, 2010. " Censo 2010", http://censo2010.ibge.gov.br/ ultimo accesso febbraio 2014.

ICO, 2002." The global coffee crisis: a Threat to sustainable development", International Coffee Organization.Submission to the World Summit on Sustianble

Development,Johannesburg, 2002.

ICO, 2003."Impact of the coffee crisis on poverty in producing countries", International Coffee Organization, ICC, Rev.1., 89-5.

ICO, 2004."Lessons from the world coffee crisis: A serious problem for sustainable development", International Coffee Organization.Executive Director's submission to UNCTAD 11th Conference.

ICO, 2009."Coffee price volatility", International Coffee Organization, 103rd International Coffee Council.

ICO, 2010. "Cyclic patterns in the supply of coffee", ICC 105-1, Sep 2010, London.

ICO, 2011a."Coffee value chain in selected importing countries". International Coffee Council 106th session,106-1, March 2011, London.

ICO, 2011b."Relaçao entre os preços do cafè nos mercados fisico e de futuros", International Coffee Organization, ICC 107-4, Sep 2011, London.

IFAD, 2011. "Rural Poverty Report 2011. New realities, new challenges: new opportunities for tomorrow's generation. The International Fund for Agricultural Development (IFAD), Roma.

MOGUEL P., TOLEDO V., 1999. "Biodiversity conservation in traditional Coffee Systems in Mexico", Conservation Biology, Vol. 13, pp. 1121.

PEARCE D., ATKINSON G., MOURATO S., 2006. "Cost-Benefit Analysis and the Environment. RECENT DEVELOPMENTS", OECD Publishing. PERFECTO I., VANDERMEER J., Mas A., Pintoet L., 2005."Biodiversity, yield and coffee certification", Ecological Economics, vol.54, pp.435-446.

PERMAN R., MA Y., mc Gilvray J., Common M., 2003. "Natural resource and Environmental
Economics", Pearson Education Limited, Harlow, Essex (UK).

PIMBERT M.P., 2008. "Towards Food Sovereignty. Reclaiming autonomous food systems", IIED, Londra, 2009.

PRETTY J., NOBLE A.D., BOSSIO D., DIXON J., HINE R.E., PENNING DE VRIES F.W., MORRISON J.I., 2006. "Resource-Conserving agriculture increases yields in developing countries", Environmental Science and Technology, vol. 40, n. 4, pp. 1114-1119. REICHHUBER A., REQUATE T., 2012."Alternative use system for the remaining Ethiopian cloud forest and the role of Arabica coffee-A cost - benefit analysis", Ecological Economics, Vol.74, pp. 102-113.

SILVANO R., UDVARDYB S., CERONIC M., FARLEY J.,2005. "An ecological integrity assessment of a Brazil Atlantic Forest watershed based on a surverys of stream health and local farmers' perceptions: implication for management", Ecological Economics, Vol.53, pp. 369 - 385.

SOS MATA ATLANTICA, 2012. www.sosma.org.br consultato nel mese di maggio-luglio 2012.

VORLEY B. ,2003. "Food, inc. Corporate concentration from farm to consumer". IIED, Uk Food Group.

WOJTKOWSKI P., 2008. "Agroecological Economics. Sustainability and Biodiversity", ACADEMIC PRESS, Elsevier Inc. 2008, San Diego (USA). 